\title{
Revista Colombiana de

\section{Escala de Calgary para el diagnóstico del síncope vasovagal. Estudio de pruebas diagnósticas}

\author{
Jhonny A. Castaño-Morales ${ }^{a, *}$, Johan S. Lopera-Valle ${ }^{a}$, Daniel A. Vanegas-Elorza ${ }^{a}$, \\ Eliana M. Cañas-Arenas ${ }^{b}$ y Edgardo González-Rivera ${ }^{c}$
}

\author{
a Facultad de Medicina, Universidad Pontificia Bolivariana, Medellín, Antioquia, Colombia \\ b Clínica Cardio VID, Medellín, Antioquia, Colombia \\ c Cardiología y Electrofisiología, Clínica Cardio VID, Medellín, Antioquia, Colombia
}

Recibido el 27 de octubre de 2014; aceptado el 18 de marzo de 2015

Disponible en Internet el 9 de mayo de 2015

\author{
PALABRAS CLAVE \\ Síncope; \\ Sistema nervioso \\ autónomo; \\ Test; \\ Diagnóstico
}

\begin{abstract}
Resumen
Objetivo: Evaluar la utilidad de la escala de Calgary en el diagnóstico de síncope vasovagal comparada con los resultados de la prueba de mesa inclinada (Tilt test) bajo protocolo sensibilizado con medicamentos.

Metodología: Estudio prospectivo de pruebas diagnósticas en pacientes con sospecha de síncope vasovagal sometidos a prueba de mesa inclinada (Tilt test) con vasodilatación inducida con nitroglicerina por vía sublingual. Se incluyeron pacientes mayores de 18 años de edad y se excluyeron pacientes con cardiopatía estructural documentada.

Resultados: Se analizaron 100 pacientes, promedio de 48,7 años (DE 19,7), 69\% de género femenino y $4 \%$ residentes en área rural. La mediana de síncopes al momento del Tilt test fue de 5 (RIQ 2-15) con presentación del primer episodio a los 41 años (RIQ 21-57), en el 52\% de los pacientes no se identificaron desencadenantes y las manifestaciones clínicas referidas en los episodios previos al Tilt test fueron diaforesis $(58 \%)$, palidez $(55 \%)$, mareo $(70 \%)$, palpitaciones (50\%) y náuseas (42\%); durante el Tilt test se reportaron mareo (65\%), diaforesis (19\%), náuseas (18\%) y visión borrosa (18\%). Se obtuvo una sensibilidad del 77,7\% (IC 95\%: 66,7-88,8) y una especificidad del 40,5\% (IC 95\%: 23,3-57,7) para la escala de Calgary.

Conclusiones: Debido a su buena sensibilidad y fácil aplicación, la escala de Calgary es un instrumento útil para el abordaje diagnóstico de pacientes con sospecha de síncope vasovagal y corazón estructuralmente sano, especialmente en menores de 50 años de edad.

(C) 2014 Sociedad Colombiana de Cardiología y Cirugía Cardiovascular. Publicado por Elsevier España, S.L.U. Este es un artículo Open Access bajo la licencia CC BY-NC-ND (http://creativecommons.org/licenses/by-nc-nd/4.0/).
\end{abstract}

\footnotetext{
* Autor para correspondencia.

Correo electrónico: jhonny1017@hotmail.com (J.A. Castaño-Morales).
} 


\section{KEYWORDS}

Syncope; Autonomic nervous system;

Test;

Diagnosis

\section{Calgary score for the diagnosis of vasovagal syncope. Diagnostic tests study}

\begin{abstract}
Objective: Evaluate the utility of Calgary score for the diagnosis of vasovagal syncope compared with the results of the Tilt table test using sensibilized protocol with medicines.

Methodology: Prospective study of diagnostic tests in patients with suspected diagnostic of vasovagal syncope who underwent tilt table test with induced vasodilation with sublingual nitroglycerin. Patients over 18 years were included and patients with documented structural heart disease were excluded.

Results: 100 patients were analyzed, averaging 48.7 years of age (SD 19.7), 69\% of feminine genre and $4 \%$ residents in rural area. Medium of syncope at the moment of tilt table test was 5 (IQR 2-15), with presentation of first episode at 41 years (IQR 21-57), in 52\% of the patients triggers were not identified and the clinical manifestations referred in episodes previous to Tilt test were diaphoresis $(58 \%)$, pallor $(55 \%)$, dizziness $(70 \%)$, palpitations $(50 \%)$ and nausea $(42 \%)$; during the test dizziness $(65 \%)$, diaphoresis $(19 \%)$, nausea $(18 \%)$ and blurred vision $(18 \%)$ were reported. A sensitivity of $77.7 \%$ was obtained $(95 \% \mathrm{Cl} 88.866 .7)$ and specificity of $40.5 \%(95 \%$ Cl 23.3 - 57.7) for the Calgary score.

Conclusions: Due to its high sensitivity and easy application, the Calgary score is a useful diagnostic approach instrument for patients with suspected vasovagal syncope and who have a structurally healthy heart, especially in those younger than 50 years.

(c) 2014 Sociedad Colombiana de Cardiología y Cirugía Cardiovascular. Published by Elsevier España, S.L.U. This is an open access article under the CC BY-NC-ND license (http://creativecommons.org/licenses/by-nc-nd/4.0/).
\end{abstract}

\section{Introducción}

El síncope es una causa frecuente de consulta, genera alrededor del $3 \%$ de los ingresos a urgencias y del uno al seis por ciento de las hospitalizaciones, posee una incidencia acumulada durante toda la vida de hasta $35 \%$ para la población general, lo cual genera un alto costo para el sistema de salud $^{1-3}$. Se clasifica según su origen en cardiogénico, secundario a hipotensión ortostática y neuromediado, este último resultado de una disfunción transitoria en un complejo arco reflejo de los sistemas nervioso central y periférico, el cual, en estudios poblacionales constituye el tipo más frecuente de síncope y se presenta actualmente con características epidémicas $^{4-6}$.

El abordaje inicial de un paciente con síncope consiste en una historia clínica completa, un adecuado examen físico y un electrocardiograma, a partir de estos resultados se orienta el diagnóstico y se realizan exámenes complementarios, entre ellos el Tilt test, una herramienta sensible, específica y reproducible para identificar entre otras una etiología sincopal neuromediada ${ }^{7-9}$, esta prueba diagnóstica comprueba los mecanismos del sistema nervioso central sobre el sistema nervioso autónomo evaluando la respuesta compensadora del corazón y del aparato circulatorio en respuesta a los cambios posturales y de presión arterial ${ }^{10,11}$. Los protocolos de Tilt test usados comúnmente utilizan dosis incrementales de isoproterenol ${ }^{12} \mathrm{o}$ una dosis única de nitroglicerina por vía sublingual ${ }^{13}$, reportando una sensibilidad del 61-69\% y una especificidad del $92-94 \%$ para el diagnóstico de síncope reflejo (neuromediado) ${ }^{10,14}$.

La escala de Calgary por su parte, es un instrumento alternativo para el diagnóstico de síncope vasovagal en pacientes con un corazón estructuralmente sano, consta de siete preguntas enfocadas en características específicas de la historia médica, factores desencadenantes y signos o síntomas presentados por el paciente durante el probable episodio sincopal ${ }^{15-17}$. Cada respuesta afirmativa otorga un puntaje entre $-5 y+3$, esto depende de que tanto apoye o no el diagnóstico la característica evaluada, al final de la encuesta se suman y restan los puntajes obtenidos para obtener una puntuación total (rango entre $-14 \mathrm{a}+6$ ). Su interpretación se realiza de la siguiente manera: un puntaje total mayor o igual a -2 sugiere un origen vasovagal del episodio sincopal y un valor menor a -2 indica que el síncope tiene otra causa (tabla 1).

El tratamiento consiste en educar al paciente, evitar estímulos desencadenantes y expandir el volumen plasmático aumentando la ingesta de agua y sal, en los casos en que se presentan síntomas premonitorios es útil la aplicación de maniobras de contrapresión en las extremidades para incrementar la presión arterial y así mejorar el retorno venoso y la perfusión cerebral, evitando o retrasando el síncope. Los pacientes refractarios requieren medicamentos como agentes vasopresores, betabloqueadores o incluso marcapasos $^{18,19}$.

El objetivo del presente estudio fue evaluar el rendimiento diagnóstico de la escala de Calgary para síncope vasovagal comparada con la prueba de mesa basculante (Tilt test).

\section{Metodología}

Se llevó a cabo un estudio prospectivo de pruebas diagnósticas en los pacientes con sospecha de síncope vasovagal que fueron sometidos a la prueba de mesa inclinada (Tilt test) en una institución de cuarto nivel de complejidad especializada en el área cardiovascular entre junio de 2013 y 
Tabla 1 Escala de Calgary

\begin{tabular}{|c|c|}
\hline Pregunta & Puntaje \\
\hline $\begin{array}{l}\text { ¿Tiene antecedentes de al menos } \\
\text { alguna de las siguientes } \\
\text { patologías: bloqueo fascicular o } \\
\text { bifascicular, asistolia, } \\
\text { taquicardia supraventricular, } \\
\text { diabetes mellitus? }\end{array}$ & -5 \\
\hline $\begin{array}{l}\text { Cuando existieron testigos } \\
\text { durante el síncope ¿notaron que } \\
\text { se puso «azul» (cianótico)? }\end{array}$ & -4 \\
\hline $\begin{array}{l}\text { ¿Los episodios de síncope } \\
\text { comenzaron a los } 35 \text { años } \\
\text { o después de esta edad? }\end{array}$ & -3 \\
\hline $\begin{array}{l}\text { ¿Recuerda algo mientras estaba } \\
\text { inconsciente? }\end{array}$ & -2 \\
\hline $\begin{array}{l}\text { ¿Tiene mareos o desmayos cuando } \\
\text { está sentado o de pie por } \\
\text { mucho tiempo? }\end{array}$ & +1 \\
\hline $\begin{array}{l}\text { ¿Tiene sudoración o siente calor } \\
\text { antes del desmayo? }\end{array}$ & +2 \\
\hline $\begin{array}{l}\text { ¿Tiene mareos o desmayos con el } \\
\text { dolor o las maniobras médicas? }\end{array}$ & +3 \\
\hline
\end{tabular}

El puntaje solo se suma o resta si la respuesta a la pregunta es afirmativa. Un resultado total $\geq-2$ indica síncope de etiología vasovagal.

Fuente: Sheldon et al. ${ }^{15}$ y Arce et al. ${ }^{17}$.

junio de 2014, bajo el protocolo italiano sensibilizado con medicamentos (vasodilatación inducida por nitroglicerina por vía sublingual), propuesto por Bartoletti et al. en el año $2000^{13}$. Se incluyeron todos aquellos pacientes mayores de 18 años de edad, cuyo Tilt test fue solicitado como parte de un procedimiento diagnóstico de mínimo un episodio con características sincopales y que aceptaran participar voluntariamente en el estudio. Se excluyeron los pacientes con alguna cardiopatía estructural documentada.

La recolección de la información se realizó en varios momentos: el primero, previo a la ejecución del Tilt test, en el cual un miembro del personal de salud entregaba a los pacientes un documento (encuesta) autodiligenciable, que contenía una explicación breve (300 palabras) sobre los aspectos generales de la investigación, el carácter voluntario de participación, y seis de siete preguntas de la escala de Calgary que solo admitían respuestas afirmativas o negativas (sí o no), la pregunta número uno de la escala se indagó directamente de la historia clínica debido al leguaje técnico utilizado (tabla 1).

Tras evaluar el correcto diligenciamiento de la encuesta, se realizó la segunda parte de la recolección de la información a través de la revisión de la historia clínica y el reporte del Tilt test de cada paciente, de esta manera se obtuvo la información correspondiente a las variables sociodemográficas, las clínicas y las de ambas pruebas diagnósticas. Cuando fue necesario completar o corroborar la información, se contactó vía telefónica con el paciente en cuestión, utilizando un protocolo de llamada previamente diseñado.

Finalmente se desarrolló un plan de análisis utilizando los programas estadísticos IBM SPSS 19 (SPSS Inc; Chicago,
Illinois, EE. UU.) para las estadísticas descriptivas y EPIDAT 3.1 para los demás datos estadísticos.

Con los resultados de las dos pruebas diagnósticas aplicadas se calculó la sensibilidad, especificidad, valor predictivo positivo, valor predictivo negativo y prevalencia para la escala de Calgary con respecto al diagnóstico de síncope vasovagal.

La investigación obtuvo el aval por parte del comité de ética de la facultad de medicina y de la clínica en cuestión, además, se realizó con base en los principios éticos para la investigación, ciñéndose a la Declaración de Helsinki y resolución 008430 de 1993 del Ministerio de Salud de Colombia.

\section{Resultados}

Se presentan los resultados correspondientes al análisis de 100 pacientes. Con respecto a las características sociodemográficas, el promedio de edad fue de 48,7 años (DE 19,7), $70 \%$ de los pacientes se encontraban en edad productiva, $69 \%$ correspondieron al género femenino y $4 \%$ residían en área rural. Dos terceras partes de los Tilt test fueron ordenados por médicos internistas (33\%) o cardiólogos (31\%), $10 \%$ por médicos generales. El $17 \%$ de los pacientes refirió antecedente familiar, en primer grado de consanguinidad, de episodios sincopales similares al suyo. Al indagar por los antecedentes patológicos, se encontró que el $56 \%$ de los pacientes no tenían ningún antecedente y dentro de las comorbilidades reportadas, la hipertensión arterial fue la más frecuente, en tres de cada 10 pacientes, seguida por diabetes mellitus y otros diagnósticos (tabla 2 ).

La mediana de episodios sincopales experimentados por cada paciente al momento del estudio fue de cinco (RIQ 215), con una mediana de edad de presentación del primer evento de 41 (RIQ 21-57) años. Un poco más de la mitad de los pacientes (52\%), dijo no haber identificado situaciones asociadas al desencadenamiento del episodio sincopal, dentro de los desencadenantes más frecuentemente referidos se encuentran: permanecer de pie por largo tiempo o en un ambiente caluroso, en el 30 y $14 \%$ respectivamente.

Con respecto a las manifestaciones clínicas en torno al episodio sincopal, se indagaron aquellas referidas por cada paciente, encontrándose diaforesis (58\%) y palidez $(55 \%)$ como los signos más frecuentes, mientras que mareo $(70 \%)$, palpitaciones $(50 \%)$ y náuseas $(42 \%)$ fueron los síntomas que más se presentaron. Por otra parte, las manifestaciones clínicas reportadas en el informe del Tilt test fueron mareo $(65 \%)$, diaforesis $(19 \%)$, náuseas $(18 \%)$ y visión borrosa $(18 \%)$, cabe mencionar que el $28 \%$ de los pacientes no presentaron síntomas durante la prueba.

Tras la aplicación de la Escala de Calgary, se obtuvo un resultado positivo para síncope vasovagal (mayor o igual a 2 ) en el $71 \%$ de los pacientes. En la tabla 3 se puede observar el porcentaje de positividad de cada una de las preguntas de la escala, cabe resaltar que más de la mitad de los pacientes refirieron el inicio de los síncopes a una edad mayor o igual a los 35 años, seis de cada 10 presentaban mareos o síncopes tras estar sentado o de pie por mucho tiempo, y sentían además sudoración o calor antes del episodio sincopal.

Por otra parte, el Tilt test fue positivo para síncope neuralmente mediado en el 63\% (tabla 4); de estos 63 pacientes, 
Tabla 2 Características sociodemográficas y clínicas

\begin{tabular}{lc}
\hline Variables sociodemográficas & $\mathrm{n}(\%)$ \\
\hline Sexo & \\
$\quad$-Masculino & $31(31)$ \\
-Femenino & $69(69)$ \\
Edad & \\
$\quad$ - 50 años & $57(57)$ \\
->50 años & $43(43)$ \\
Ocupación & \\
-Estudiante & $7(7)$ \\
-Empleado & $55(55)$ \\
-Desempleado & $9(9)$ \\
-Jubilado & $29(29)$ \\
Procedencia & \\
-Urbano & $96(96)$ \\
-Rural & $4(4)$ \\
& \\
Variables clínicas & $\mathrm{n}(\%)$ \\
\hline Antecedente familiar de síncopes & $17(17)$ \\
Orden del Tilt test & \\
-Internista & $33(33)$ \\
-Cardiólogo & $31(31)$ \\
-Médico general & $10(10)$ \\
-Otro & $10(10)$ \\
-Desconocido & $6(6)$ \\
Comorbilidades & \\
-Hipertensión arterial & $31(31)$ \\
-Biabetes mellitus & $9(9)$ \\
-Taquicardia supraventricular & $1(1)$ \\
-Otros & $1(1)$ \\
\hline & $9(9)$ \\
\hline
\end{tabular}

Tabla 3 Porcentaje de positividad de las preguntas de la Escala de Calgary

\begin{tabular}{lll}
\hline Pregunta & Puntaje & Sí (\%) \\
\hline $\begin{array}{l}\text { Antecedente de: bloqueo } \\
\text { fascicular o bifascicular, } \\
\text { asistolia, taquicardia } \\
\text { supraventricular o diabetes } \\
\quad \text { mellitus }\end{array}$ & -5 & 11 \\
$\begin{array}{l}\text { Cianosis durante el síncope } \\
\text { Inicio de los síncopes a los }\end{array}$ & -4 & \\
$\quad \begin{array}{l}35 \text { o más años de edad } \\
\text { Recuerdo durante la } \\
\text { inconsciencia }\end{array}$ & -3 & 7 \\
$\begin{array}{l}\text { Mareos o síncopes cuando está } \\
\text { sentado o de pie por mucho } \\
\text { tiempo }\end{array}$ & -2 & 55 \\
$\begin{array}{l}\text { Sudoración o calor antes del } \\
\text { síncope }\end{array}$ & +2 & 61 \\
$\begin{array}{l}\text { Mareos o síncopes con el dolor } \\
\text { o las maniobras médicas }\end{array}$ & +3 & 60 \\
\hline
\end{tabular}

Tabla 4 Comparación de la Escala de Calgary vs. Tilt test para diagnóstico de síncope neuralmente mediado

\begin{tabular}{lllr}
\hline Escala Calgary & \multicolumn{2}{c}{ Tilt test } & Total \\
\cline { 2 - 3 } & Positivo & Negativo & \\
\hline Positivo $(\geq-2)$ & 49 & 22 & 71 \\
Negativo & 14 & 15 & 29 \\
Total & 63 & 37 & 100 \\
\hline
\end{tabular}

Tabla 5 Rendimiento diagnóstico de la Escala de Calgary para síncope neuralmente mediado

\begin{tabular}{lrrr}
\hline Estadístico & Valor & \multicolumn{2}{c}{ IC 95\% } \\
\hline Sensibilidad (\%) & 77,78 & 66,72 & 88,84 \\
Especificidad (\%) & 40,54 & 23,37 & 57,71 \\
VPP" & 69,01 & 57,55 & 80,47 \\
VPN $^{\text {a }}$ & 51,72 & 31,81 & 71,64 \\
Prevalencia (\%) & 63 & 53,04 & 72,96 \\
\hline
\end{tabular}

"Valor predictivo positivo.

a Valor predictivo negativo.

9 de cada 10 experimentaron el síncope en la fase tres o fase de provocación farmacológica con nitroglicerina sublingual. La media del tiempo transcurrido del examen hasta la producción del síncope fue de 30,4 (DE 3,9) minutos y las medianas de la presión arterial sistólica y diastólica, así como la frecuencia cardiaca registradas justo durante el síncope fueron de 45 (RIQ 42-50) mmHg, 28 (RIQ 21-33) mmHg y 62 (40-80) latidos por minuto, respectivamente.

Así pues, tras realizar la evaluación del rendimiento de la escala de Calgary como prueba diagnóstica y en comparación con el Tilt test, para el diagnóstico de síncope neuralmente mediado se obtuvo una sensibilidad del 77,7\% (IC 95\%: 66,788,8 ) y una especificidad del $40,5 \%$ (IC 95\%: $23,3-57,7$ ), el valor predictivo positivo fue del $69 \%$ con un valor predictivo negativo del 51\% (tabla 5).

Dividiendo el análisis por género y edad mayor o menor de 50 años se obtuvieron los siguientes resultados: hombres ( $\mathrm{n}$ : 31, sensibilidad: 72,2 , especificidad: 61,5$)$, mujeres (n: 69, sensibilidad: 80 , especificidad: 29,1), menores de 50 años (n: 57, sensibilidad: 94,2, especificidad: 31,8), mayores de 50 años (n: 43, sensibilidad: 57,1, especificidad: 53,3).

\section{Discusión}

El Tilt test es una prueba diagnóstica para síncope vasovagal frecuentemente usada en nuestro medio, pero a pesar de ser un procedimiento seguro y con baja frecuencia de complicaciones ${ }^{20}$, es una prueba que por su valor, características y requerimientos; tales como la disponibilidad de mesa basculante en un servicio especializado, evaluación por cardiología y alto costo económico, no está disponible para toda la población y además resulta incómodo para los pacientes reproducir el episodio sincopal con todos sus signos y síntomas bajo condiciones controladas ${ }^{10,13,21}$. Es por ello que la escala de Calgary se proyecta como un instrumento con gran utilidad en situaciones en las que se cuenta con recursos logísticos y económicos limitados. 
En el estudio original desarrollado por Sheldon et al. con el fin de proponer la escala de Calgary a la comunidad científica en el año 2006, se logró clasificar correctamente el $90 \%$ de los pacientes cuidadosamente seleccionados, reportando $89 \%$ de sensibilidad y $91 \%$ de especificidad para el diagnóstico de síncope vasovagal ${ }^{15}$. Con estos hallazgos se planteó entonces la posibilidad de usar la escala como un instrumento con excelente rendimiento diagnóstico para síncope vasovagal, teniendo en cuenta su fácil aplicabilidad y costo-efectividad. Posteriormente Romme et al. realizaron un estudio en Holanda (seguimiento de 380 pacientes por dos años) para evaluar el rendimiento de la escala y obtuvieron una sensibilidad de $87 \%$ y una especificidad de $32 \%{ }^{16}$. Además, se han efectuado otros estudios para evaluar la utilidad de la escala en poblaciones con características específicas, por ejemplo en un estudio publicado por Expósito et al. evaluaron el rendimiento diagnóstico de la escala de Calgary en pacientes mayores con alteraciones cardiacas leves reportando sensibilidad de $51 \%$ y especificidad de $73 \% 22$.

Con respecto al rendimiento obtenido de la escala de Calgary en el presente estudio (77\% de sensibilidad y $40 \%$ de especificidad), aunque el valor de sensibilidad es el más bajo obtenido de los estudios publicados hasta ahora, sigue siendo importante y útil, pudiendo llegar a ser tan alto como del $88,8 \%$. En general el rendimiento de la escala fue muy homogéneo en los diferentes grupos en los que se realizó el análisis, la mejor sensibilidad se obtuvo en los pacientes de género femenino y en menores de 50 años de edad, comparados con el grupo de pacientes mayores de 50 años de edad en los que probablemente el sesgo de memoria fue influyente al responder las preguntas de la escala y por ello presentaron la menor sensibilidad, lo que concuerda con los resultados publicados por Expósito et al. corroborando la hipótesis sobre el menor rendimiento diagnóstico de la escala de Calgary en pacientes mayores que no pueden recordar muy bien las características del episodio sincopal ${ }^{22}$.

Además, se deben tener en cuenta algunas características en el diseño de los estudios precedentes que marcan diferencias con respecto a la metodología y los resultados del presente estudio, por ejemplo en el estudio realizado por Romme et al. se llevó a cabo un seguimiento clínico por dos años junto con la realización de otras pruebas adicionales, lo que podría dar una razón a la mayor sensibilidad reportada. Del otro lado, se confirma la especificidad reportada por Romme et al. (32\%) que según los resultados mencionados previamente podría llegar a ser tan baja como del $23,3 \%$ y que no concuerda con el estudio original publicado por Sheldon et al. en el que seguramente se haya obtenido una especificidad tan alta $(91 \%)$ debido a la minuciosa selección bajo estrechos criterios de elegibilidad del grupo de pacientes en el que se desarrolló la escala por parte de una unidad especializada en el diagnóstico y manejo de síncope. Cabe resaltar que las diferencias en cuanto a las características de la población estudiada pueden repercutir en el rendimiento diagnóstico de una prueba que originalmente fue diseñada para otro tipo de población, lo que plantea la posibilidad de que se desarrolle una escala de Calgary modificada y ajustada según las características de la población a estudiar.

De esta manera se puede inferir que la escala de Calgary posee una buena capacidad para captar los pacientes que presentan síncope vasovagal, sin embargo, presenta deficiencia para descartar a aquellos pacientes sanos o con otra etiología, expresado de otra forma; la buena sensibilidad de la escala permite captar gran cantidad de pacientes, pero no la adecuada caracterización de estos, que pueden padecer síncopes de etiología neuromediada versus cardiaca, o ninguna de ellas. En cuanto a los valores predictivos del presente estudio, el más promisorio parece ser el valor predictivo positivo que puede llegar a ser de hasta $80,4 \%$, indicando que la posible causa etiológica del síncope sea vasovagal y que según la disponibilidad de recursos y criterio clínico valdría la pena confirmarlo solicitando un Tilt test.

En el estudio más cercano al contexto latinoamericano, llevado a cabo en Argentina por Arce et al. se determinó la utilidad del Tilt test no sensibilizado con medicamentos en 70 pacientes mayores de 15 años de edad usando la escala de Calgary para obtener la probabilidad pretest de síncope vasovagal. Los autores concluyeron que en pacientes con síncope vasovagal sugerido por la evaluación clínica, la prueba de inclinación sin sensibilización por fármacos no proporcionó información adicional para el diagnóstico, y que la escala de Calgary constituye un instrumento orientador útil en la mayoría de los $\operatorname{casos}^{17}$.

Con respecto a las características sociodemográficas de la población estudiada, en general se tuvo una muestra que corresponde con lo descrito en la literatura, confirmando que el síncope vasovagal es una patología más frecuente en el género femenino y sus distribución por edad es muy variable, siendo más fácilmente diagnosticable en personas jóvenes sin otros antecedentes patológicos ${ }^{17,23,24}$. Al verificar la gran prevalencia de esta patología en la población productiva se confirma el impacto que tiene con respecto a las incapacidades, el tiempo laboral perdido y la calidad de vida, por ello la importancia de su sospecha, el diagnóstico y el tratamiento precoz ${ }^{24,25}$. En cuanto a la distribución geográfica de la población, el predominio de pacientes residentes en el área urbana apoya la teoría de que probablemente el traslado y los recursos de un habitante del área rural sea más difícil y por tanto pueda ser útil enfocar el diagnóstico con la escala de Calgary para tomar una decisión con respecto a solicitar o no el Tilt test u otros exámenes complementarios.

La edad de inicio de los episodios suele ser muy variable, desde jóvenes e incluso niños hasta ancianos, en estos últimos se deben tener en cuenta otras posibles causas ${ }^{26,27}$. El promedio de episodios previos a la realización del Tilt test fueron pocos, ya que en general estos episodios suelen ser muy sintomáticos y preocupan mucho al paciente y sus familiares, por tanto hay que sospechar que los relatos de múltiples episodios corresponden a pacientes con mucho tiempo de evolución o no corresponden a síncope vasovagal. En la literatura se ha planteado la posible predisposición genética a padecer síncope vasovagal, se deja el $17 \%$ de pacientes que refirió dicho antecedente en este estudio como un dato estadístico más para la discusión ${ }^{28-30}$.

Es de vital importancia ser enfático en el interrogatorio en el momento de preguntar por la pérdida de la consciencia ya que los pacientes suelen interpretar como síncope cualquier episodio en el que se pierda el tono postural más no el conocimiento y una respuesta negativa aleja mucho la probabilidad diagnóstica del síncope vasovagal. También 
es mandatorio indagar por los desencadenantes del síncope, y aunque entre estos pacientes la respuesta más frecuente fue no haberse percatado de ningún desencadenante, permanecer de pie por largo tiempo o en un ambiente caluroso siguen siendo las respuestas positivas más comunes ${ }^{21,31,32}$.

Con respecto a las manifestaciones clínicas, estas correspondieron con lo reportado en la literatura (diaforesis, náuseas, visión borrosa, cefalea, palpitaciones, entre otras), existiendo una clara correlación entre la clínica manifestada en los episodios antes de la prueba por parte de los pacientes y en las manifestaciones reportadas en el Tilt test, por tanto un interrogatorio bien dirigido y ejecutado podría equipararse a realizar un Tilt test, esto en cuanto al cuadro clínico sugestivo. Luego habría que investigar sobre el valor predictivo positivo de los signos y síntomas más comunes para esta patología 2,21,33.

En el contexto actual, la solicitud del Tilt test tiende a ser un examen solicitado por especialistas, en ese caso la escala de Calgary se posiciona como un instrumento útil para los médicos generales; previo a la solicitud de un Tilt test, antes de buscar otras posibles etiologías o remitir a un especialista.

\section{Limitaciones}

Aunque se obtuvo una muestra representativa de pacientes de todas la edades, el $75 \%$ de la población estudiada fue mayor de 31 años de edad, teniendo en cuenta las implicaciones y manifestaciones tempranas del síncope vasovagal, es de gran importancia para futuros estudios delimitar más la población con el fin de evaluar el impacto y desempeño de la escala en la población más joven. Esta patología también tiene relevancia en la población pediátrica, la cual no se incluyó en el diseño de este estudio.

Se requieren realizar análisis más rigurosos con una muestra poblacional mayor y más seleccionada para poder brindar conclusiones de mayor peso y validez estadística. Por último, a pesar del desarrollo y validación de la escala de Calgary en países como Canadá y Holanda, sigue siendo prioritario llevar a cabo su validación en países de habla hispana haciendo una traducción más precisa y confiable.

\section{Conclusión}

Debido a su buena sensibilidad, la cual se ha podido reproducir en este y otros estudios, y a su fácil aplicación, la escala de Calgary es un instrumento útil en determinados contextos para el abordaje diagnóstico y enfoque inicial de los pacientes con sospecha de síncope vasovagal y corazón estructuralmente sano, pero ante un resultado positivo no deben descartarse otras posibles causas y es necesario hacer uso de otros elementos diagnósticos para confirmar la etiología sincopal, especialmente en la población mayor de 50 años de edad.

\section{Responsabilidades éticas}

Protección de personas y animales. Los autores declaran que para esta investigación no se han realizado experimentos en seres humanos ni en animales.
Confidencialidad de los datos. Los autores declaran que han seguido los protocolos de su centro de trabajo sobre la publicación de datos de pacientes.

Derecho a la privacidad y consentimiento informado. Los autores han obtenido el consentimiento informado de los pacientes y/o sujetos referidos en el artículo. Este documento obra en poder del autor de correspondencia.

\section{Conflicto de intereses}

Los autores declaran no tener ningún conflicto de intereses.

\section{Agradecimientos}

Al semillero de investigación SIFAM de la Universidad Pontificia Bolivariana y a la Clínica Cardio VID por apoyar la formación en investigación y facilitar la captación de los pacientes.

\section{Bibliografía}

1. Hatoum T, Sheldon R. A practical approach to investigation of syncope. Can J Cardiol. 2014;30:671-4.

2. Chen-Scarabelli C, Scarabelli TM. Neurocardiogenic syncope. BMJ. 2004;329:336-41.

3. Saklani P, Krahn A, Klein G. Syncope. Circulation. 2013;127: 1330-9.

4. Moya-I-Mitjans Á, Rivas-Gándara N, Sarrias-Mercè A, PérezRodón J, Roca-Luque I. Syncope. Rev Esp Cardiol (Engl Ed). 2012;65:755-65.

5. Fu Q, Levine BD. Pathophysiology of neurally mediated syncope: Role of cardiac output and total peripheral resistance. Auton Neurosci Basic Clin. 2014;184:24-6.

6. Hainsworth R. Pathophysiology of syncope. Clin Auton Res. 2004;14:i18-24.

7. Blanc JJ. Clinical laboratory testing: what is the role of tilt-table testing, active standing test, carotid massage, electrophysiological testing and ATP test in the syncope evaluation. Prog Cardiovasc Dis. 2013;55:418-24.

8. Brignole M, Menozzi C, Rosso AD, Costa S, Gaggioli G, Bottoni $\mathrm{N}$, et al. New classification of haemodynamics of vasovagal syncope: beyond the VASIS classification. Europace. 2000;2:66-76.

9. Sandhu KS, Khan P, Panting J, Nadar S. Tilt-table test: its role in modern practice. Clin Med Lond Engl. 2013;13:227-32.

10. Moya A, Sutton R, Ammirati F, Blanc JJ, Brignole M, Dahm JB, et al. Guidelines for the diagnosis and management of syncope. Eur Heart J. 2009;30:2631-71.

11. Carey BJ, Manktelow BN, Panerai RB, Potter JF. Cerebral autoregulatory responses to head-up tilt in normal subjects and patients with recurrent vasovagal syncope. Circulation. 2001;104:898-902.

12. Morillo CA, Klein GJ, Zandri S, Yee R. Diagnostic accuracy of a low-dose isoproterenol head-up tilt protocol. Am Heart J. 1995; 129:901-6.

13. Bartoletti A, Alboni P, Ammirati F, Brignole M, Rosso AD, Manzillo GF, et al. 'The Italian Protocol': a simplified head-up tilt testing potentiated with oral nitroglycerin to assess patients with unexplained syncope. Europace. 2000;2:339-42.

14. Forleo C, Guida P, lacoviello M, Resta M, Monitillo F, Sorrentino $\mathrm{S}$, et al. Head-up tilt testing for diagnosing vasovagal syncope: a meta-analysis. Int J Cardiol. 2013;169:e49-50. 
15. Sheldon R, Rose S, Connolly S, Ritchie D, Koshman ML, Frenneaux M. Diagnostic criteria for vasovagal syncope based on a quantitative history. Eur Heart J. 2006;27:344-50.

16. Romme JJ, van Dijk N, Boer KR, Bossuyt PM, Wieling W, Reitsma JB. Diagnosing vasovagal syncope based on quantitative historytaking: validation of the Calgary syncope symptom score. Eur Heart J. 2009;30:2888-96.

17. Arce M, Femenía F, Palazzolo J, Trucco E, Uribe W, Baranchuk A. Es necesaria la prueba de inclinación en pacientes con diagnóstico clínico de síncope vasovagal? Resultados utilizando un protocolo no sensibilizado. Arch Cardiol México. 2011;81:287-91.

18. Guzman JC, Armaganijan LV, Morillo CA. Treatment of neurally mediated reflex syncope. Cardiol Clin. 2013;31: 123-9.

19. Kuriachan V, Sheldon RS, Platonov M. Evidence-based treatment for vasovagal syncope. Heart Rhythm Off J Heart Rhythm Soc. 2008;5:1609-14.

20. Sutton $\mathrm{R}$. The value of tilt testing and autonomic nervous system assessment. Card Electrophysiol Clin. 2013;5:403-6.

21. Grubb BP. Neurocardiogenic syncope. N Engl J Med. 2005;352:1004-10.

22. Expósito V, Guzmán JC, Orava M, Armaganijan L, Morillo CA. Usefulness of the Calgary syncope symptom score for the diagnosis of vasovagal syncope in the elderly. Europace. 2013;15:1210-4.

23. Gauer RL. Evaluation of syncope. Am Fam Physician. 2011;84: 640-50.
24. Kenny RA, Bhangu J, King-Kallimanis BL. Epidemiology of syncope/collapse in younger and older western patient populations. Prog Cardiovasc Dis. 2013;55:357-63.

25. Raj SR, Coffin ST. Medical therapy and physical maneuvers in the treatment of the vasovagal syncope and orthostatic hypotension. Prog Cardiovasc Dis. 2013;55:425-33.

26. Stewart JM. Common syndromes of orthostatic intolerance. Pediatrics. 2013;131:968-80.

27. Cooke J, Carew S, Costelloe A, Sheehy T, Quinn C, Lyons D. The changing face of orthostatic and neurocardiogenic syncope with age. QJM Mon J Assoc Physicians. 2011;104:689-95.

28. Bizios AS, Sheldon RS. Vasovagal syncope: state or trait. Curr Opin Cardiol. 2009;24:68-73.

29. Holmegard HN, Benn M, Kaijer M, Haunsø S, Mehlsen J. Prevalence of family history in patients with reflex syncope. J Clin Neurosci Off J Neurosurg Soc Australas. 2013;20:692-6.

30. Olde Nordkamp LR, Wieling W, Zwinderman AH, Wilde AA, van Dijk N. Genetic aspects of vasovagal syncope: a systematic review of current evidence. Europace. 2009;11:414-20.

31. Ouyang $H$, Quinn J. Diagnosis and evaluation of syncope in the emergency department. Emerg Med Clin North Am. 2010;28:471-85.

32. Medow MS, Stewart JM, Sanyal S, Mumtaz A, Sica D, Frishman WH. Pathophysiology, diagnosis, and treatment of orthostatic hypotension and vasovagal syncope. Cardiol Rev. 2008;16:4-20.

33. Kossaify A, Kallab K. Neurocardiogenic syncope and associated conditions: insight into autonomic nervous system dysfunction. Turk Kardiyol Dern Ars. 2013;41:75-83. 